\title{
The Missing Inferior Loop
}

\author{
Aberrant Ansa Cervicalis \\ Discovered During Left Recurrent \\ Laryngeal Re-Innervation
}

Kristian M. Black, BS ${ }^{1}$; Jennifer F. Ha, MD²; David J. Brown, MD ${ }^{1,2}$

\section{ABSTRACT}

Patent ductus arteriosus (PDA) occurs in $40-60 \%$ of preterm infants. ${ }^{1}$ Corrective surgery risks iatrogenic unilateral vocal cord paralysis (VCP) due to the anatomical position of the left recurrent laryngeal nerve (RLN). latrogenic VCP resulting from PDA ligation has an incidence of $11.5 \%,{ }^{8}$ causing debilitating speech patterns and risk for aspiration. Correction of iatrogenic damage to the RLN in pediatric patients is often achieved through laryngeal reinnervation using the ansa cervicalis (AC). The implications of anatomical AC variants on surgical outcomes have not been well studied.

We present the case of a 3-year-old boy who was referred to our institution for dysphonia secondary to a left VCP, a complication of a PDA ligation performed at an outside institution. During the operation, the AC was found to be aberrant, consisting of a single superior root arising anterior to the internal jugular vein (IJV) and deep to the common facial vein (CFV), and lacking both an inferior root and loop. Given this aberration, due to its favorable bulk and length, this patient's omohyoid branch was isolated, prepared, and used for reinnervation.

In RLN reinnervation with the $A C$, it is important to consider the variation in AC morphology and which nerve branch to select for reinnervation. Precise anatomical descriptions of the AC and its variants are important to allow surgeons to identify the best donor nerve for RLN reinnervation and correction of VCP. The lack of an inferior root in this patient had no negative clinical consequences at 18 months. Based on these outcomes, the omohyoid branch of the AC appears to be an effective donating branch option for RLN reinnervation in the setting of an absent AC inferior root. 


\section{Introduction}

Patent ductus arteriosus (PDA) occurs in 40-60\% of preterm infants. ${ }^{1}$ The recurrent laryngeal nerve (RLN) loops inferiorly around both the congenital shunt and aortic arch and then ascends to innervate the left vocal cord. A 10-year retrospective study of 19 United States children's hospitals demonstrated that 5719 (42\%) of 13853 newborns were diagnosed with PDA; of those infants with PDA, 3889 (68\%) underwent medical intervention consisting of indomethacin (54.9\%), ibuprofen (10.5\%), or both (2.6\%), while 5.6\% underwent PDA ligation as a primary intervention. ${ }^{5}$ Medical intervention alone was insufficient in 1260 (22\%) patients; in these cases, infants subsequently underwent PDA ligation. ${ }^{5}$ PDA ligation is necessary when medical therapy fails but risks iatrogenic unilateral vocal cord paralysis (VCP) due to the anatomical position of the left RLN. For patients who have experienced this iatrogenic complication and are symptomatic, one of the rehabilitative options includes laryngeal reinnervation with the ansa cervicalis (AC).

The AC is a loop of nerves innervating the superior and inferior belly of the omohyoid, sternohyoid, and sternothryoid muscles. These muscles are responsible for depressing the hyoid and larynx during phonation and swallowing. Frequently used in laryngeal reinnervation, the $A C$ 's function and location are well known, and many anatomical deviations have been described in the literature. The most common AC variant is described as a looping nerve structure, comprised of a superior and inferior root, present anterior to the internal jugular vein (IJV). ${ }^{8}$ The superior root receives nerve fibers from spinal nerve C1, descending medial to the IJV from the hypoglossal nerve. Branches from anterior rami of $\mathrm{C} 2$ and $\mathrm{C} 3$ emerge posterior to the IJV and combine to form the inferior root before descending laterally along the vessel. Though several cadaveric studies have indicated additional contribution to the inferior root from spinal nerves C1-C4, the combination C2 and C3 is observed $63-81 \%$ of the time. ${ }^{3,8}$

The implications of anatomical AC variants on surgical outcomes in laryngeal innervation have not been well studied. Though anatomical variance is commonly noted in AC morphology, to our knowledge, this is the first case report of a completely absent AC inferior root in a live patient and thus more informative than cadaveric studies in terms of discussing surgical outcomes. Reports of aberrant nerves encountered are important to head and neck surgeons because damage to donor nerves in this area can impact successful surgical outcomes. This case report describes the anatomy of an aberrant AC found arising anterior to the IJV, deep to the common facial vein (CFV), and with an absent inferior root during a left RLN reinnervation.

\section{Case Presentation}

A 3-year-old boy was referred to our institution for dysphonia secondary to left VCP, a complication of a PDA ligation performed at an outside institution during his infancy. He was born at 27 weeks due to preterm labor of a twin pregnancy with a breech presentation, requiring an emergency caesarean section. His APGAR scores were 3, 4, 7, and 8 at 1, 5, 10 , and 15 minutes, respectively. He had a complicated medical history including bronchopulmonary dysplasia, retinopathy of prematurity, resolving patent foramen ovale, necrotizing enterocolitis complicated by small bowel perforation that required resection, obstructive sleep apnea treated with adneotonsillectomy, and tympanostomy tube placement for eustachian tube dysfunction. There was no history of aspiration or recurrent pneumonia but on exam the patient was dysphonic with a weak voice.

Flexible nasoendoscopies (FNE) performed when the patient was 12 and 24 months old showed normal right true vocal cord mobility and a left atrophic vocal cord in the paramedian with absent mobility (Figure 1). These signs in the setting of a previous PDA ligation were indicative of idiopathic unilateral vocal cord paralysis, and he was deemed an appropriate candidate for left RLN reinnervation with the $A C$ and simultaneous vocal cord injection with Prolaryn ${ }^{\mathrm{TM}}$ (Merz North America, Inc.) to provide bulk to atrophied vocal cords, allowing complete closure of the gap during phonation. 
It is often used in adjunct with RLN reinnervation as the recuperation period of neural anastomoses takes several months to provide optimal vocal cord tone. There are no other alternative treatments for VCP at this age. Speech therapy cannot correct the physical abnormality, and the patient was too young for thyroplasty.

The RLN reinnervation proceeded as follows: A horizontal incision was placed to the left of the midline at the level of the cricoid cartilage. Subplatysmal flaps were raised, and the omohyoid muscle, sternocleidomastoid muscle, and IJV were identified. The IJV was dissected almost to its entirety, and the AC was not found. Instead, the AC was found anterior to the IJV and deep to the CFV (Figure 2). After finding the $A C$, it was noted to be missing an inferior root. Stimulation of the strap muscles with a handheld nerve simulator confirmed the identity of the nerve structure. The omohyoid branch of the AC was then isolated and prepared for reinnervation. The ipsilateral RLN was identified, isolated, and ligated to the AC using end-to-end perineural anastomosis under microscopic guidance.

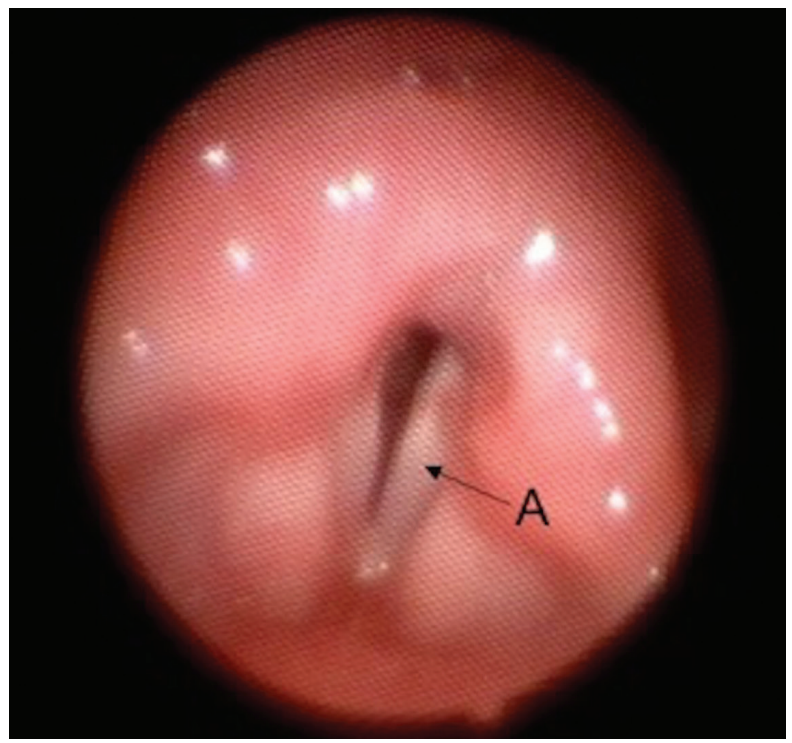

FIGURE 1. Atrophic left vocal fold (A) fixed in a paramedian position.

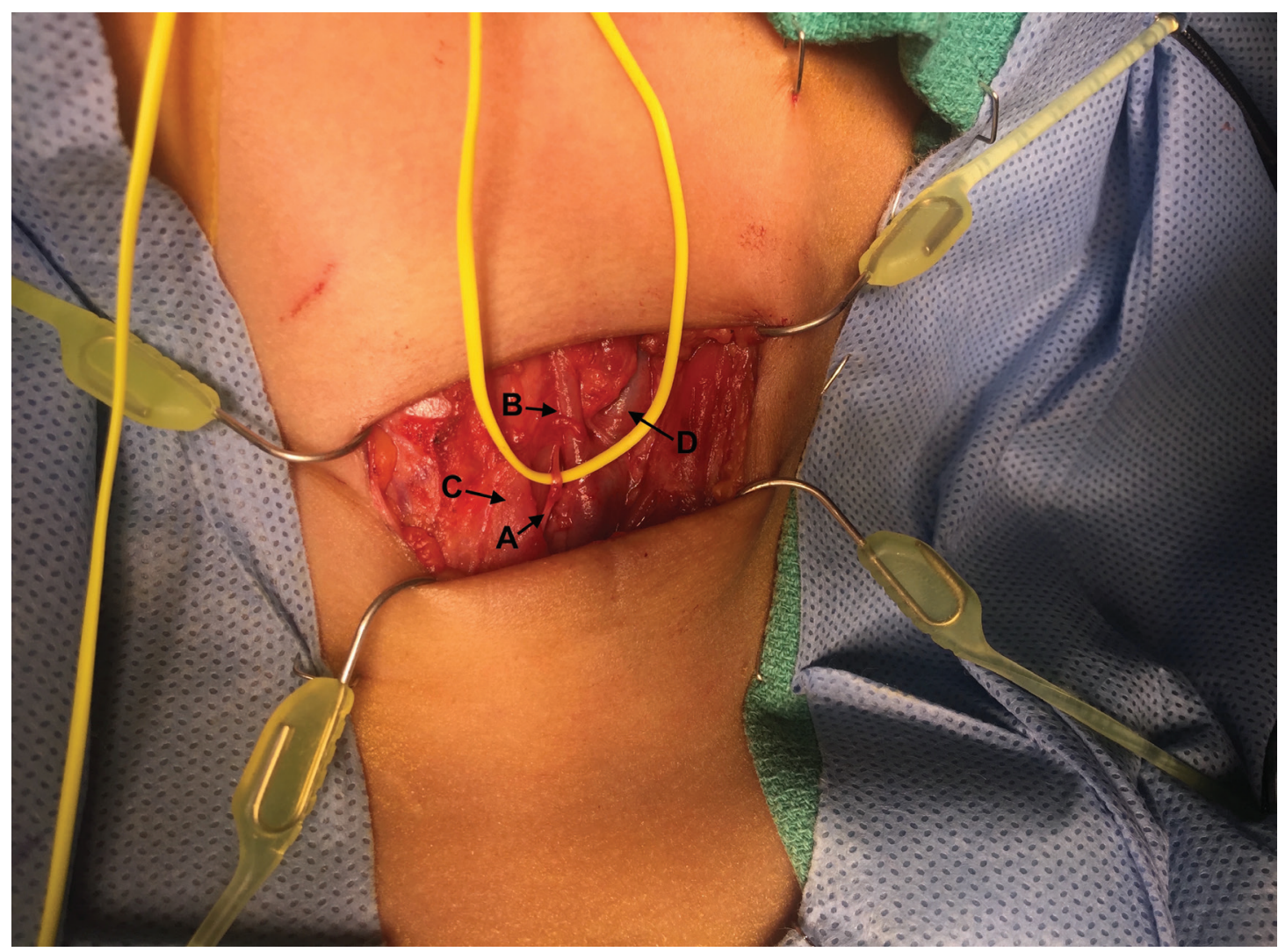

FIGURE 2. Intraoperative photograph showing the superior loop of the left AC (A) arising anterior to the IJV (D) at the bifurcation of the common facial vein (B) running deep to the strap muscles (C). 
After identification of the AC, the surgery was completed without complications, and the patient was admitted overnight for observation. There were no immediate postoperative complications, and the patient was discharged on post-op day one. He was followed postoperatively for 18 months and had significant improvement in his symptoms. His vocal quality was notable for increased clarity and projection and the absence of dysphonia and stridor.

\section{Discussion}

Precise anatomical description of the AC and its atypical morphologies allow surgeons to properly recognize these variant nerve structures and avoid iatrogenic injury. The unexpected variation found in this patient prompted a literature search on such occurrences. Cadaveric studies have reported frequencies of absent inferior roots around $3 \%$; to the best of our knowledge, this is the first case report that describes the absence of the AC inferior root in a live patient.

latrogenic VCP resulting from PDA ligation has an estimated incidence of $11.5 \% .^{7}$ Implications of this include debilitating speech patterns and an increased aspiration risk. Options for surgical correction of unilateral VCP include injection laryngoplasty, thyroplasty, and laryngeal reinnervation. Though thyroplasty is a viable option for children experiencing consistent aspiration, it is less efficient than laryngoplasty and laryngeal reinnervation at correcting dysphonia. ${ }^{4}$ According to a 2015 report comparing postoperative results of injection laryngoplasty to laryngeal reinnervation in children (age 2-16 years), reinnervation provided better perceptual and acoustic parameters. ${ }^{9}$

In RLN reinnervation with the $A C$, it is important to consider the variation in AC morphology and which nerve branch to select for reinnervation. The combination of the inferior and superior roots, carrying ascending and descending nerve fibers, creates the AC loop. From the loop emerge the segmental branches to the sternothyroid, sternohyoid, and omohyoid muscles. Notably, each branch varies in its neural fiber composition. ${ }^{2}$ The variation in composition of ascending and descending fibers gives rise to different levels of neural activity during respiration and phonation. ${ }^{6}$ Thus the best nerve for reinnervation is the one with the most selective activity during phonation. This has implications for surgical clinical decision making, particularly in the face of variant anatomy. The AC loop branch innervating the sternohyoid is an appealing candidate due to its activity during phonation and diminished phasic activity during respiration. If unavailable, however, the sternothyroid and omohyoid branches can be used effectively. ${ }^{6}$ In addition to activity, the size of the donating nerve is an important consideration. Larger nerves contain more fibers and subsequently provide more neural stimulation to the atrophied muscle when reinnervated. This allows the paralyzed vocal cord to meet with the unaffected cord at midline, greatly improving vocal quality. Due to its favorable bulk and length, this patient's omohyoid branch was selected for reinnervation, conferring the best prognosis of recovering vocal cord tone.

The lack of an AC inferior root did not impose a direct limitation on this case as it is seldom used for anastomoses in RLN reinnervation. However, it was unknown if the lack of an inferior root and its donor nerve fibers would result in an altered nerve fiber composition in the omohyoid branch that could then affect surgical outcomes. Noting the improved vocal quality, clarity, and projection, as well as this patient's recovered vocal cord tone and position at the midline, the absence of an inferior root and the use of the omohyoid branch for reinnervation appears to have had no significant clinical impact for this patient. It will be important to follow this patient and monitor for recurring symptoms of dysphonia.

\section{Conclusion}

To our knowledge, this is the first report of an RLN reinnervation using an AC with an absent inferior root. The case is noteworthy since nerve activity is a function of nerve fiber, size, and composition, and the $A C$ is composed of various nerve fibers 
from the superior and inferior roots. Studies of the $A C$ in cadavers provide extensive data on anatomical formations, but case reports detailing aberrant formations in live patients are needed to generate data in postoperative and long-term clinical outcomes. The lack of an inferior root in this patient had no negative clinical consequences at 18 months. Based on these outcomes, the omohyoid branch of the AC appears to be an effective donating branch option for RLN reinnervation in the setting of an absent AC inferior root.

\section{References}

1. Abdel-Hady H, Nasef N, Shabaan AE, Nour I. Patent Ductus Arteriosus in preterm infants: do we have the right answers? BioMed Res Int. 2013:1-15.

2. Banneheka S. Anatomy of the ansa cervicalis: nerve fiber analysis. Anat Sci Int. 2008; 83(2):61-67. Web.

3. Banneheka S. Morphological study of the ansa cervicalis and the phrenic nerve. Anat Sci Int. 2008;83(1):31-44. Web. February 3, 2016.
4. Butskiy O, Bhavik M, and Neil K. Chadha. "Surgical Interventions for Pediatric Unilateral Vocal Cord Paralysis." JAMA Otolaryngo/_Head Neck Surg. 2015;141(7):654. Web.

5. Hagadorn JI, Brownell EA, Trzaski JM, et al. Trends and variation in management and outcomes of very low-birth-weight infants with patent ductus arteriosus. Ped Res. 2016. Web. November 4, 2016.

6. Paniello RC. Laryngeal reinnervation. Vocal Fold Paralysis. 2004;37:189-202. Web.

7. Pereira K, Webb B, Blakely M, Cox C, Lally K. Sequelae of recurrent laryngeal nerve injury after patent ductus arteriosus ligation. JAMA Otolaryngo/_Head Neck Surg. 2005;133(2).

8. Mwachaka PM, Ranketi SS, Elbusaidy H, Ogeng'o J. Variations in the anatomy of ansa cervicalis. Folia Morph. 2010;69(3):160-163.

9. Zur KB, Carroll LM. Recurrent laryngeal nerve reinnervation in children: acoustic and endoscopic characteristics preintervention and postintervention. a comparison of treatment options. The Laryngoscope. 2005;125. 